\title{
Abstract: 3D Catheter Guidance Including Shape Sensing for Endovascular Navigation
}

\author{
Sonja Jäckle ${ }^{1}$, Verónica García-Vázquez ${ }^{2}$, Felix von Haxthausen ${ }^{2}$, \\ Tim Eixmann ${ }^{3}$, Malte Maria Sieren ${ }^{4}$, Hinnerk Schulz-Hildebrandt ${ }^{3,5,6}$, \\ Gereon Hüttmann ${ }^{3,5,6}$, Floris Ernst ${ }^{2}$, Markus Kleemann ${ }^{7}$, Torben Pätz ${ }^{8}$ \\ ${ }^{1}$ Fraunhofer MEVIS, Institute for Digital Medicine, Lübeck, Germany \\ ${ }^{2}$ Institute for Robotics and Cognitive Systems, Universität zu Lübeck, Germany \\ ${ }^{3}$ Medizinisches Laserzentrum Lübeck GmbH, Lübeck, Germany \\ ${ }^{4}$ Department for Radiology and Nuclear Medicine, UKSH, Lübeck, Germany \\ ${ }^{5}$ Institute of Biomedical Optics, Universität zu Lübeck, Germany \\ ${ }^{6}$ German Center for Lung Research, DZL, Großhansdorf, Germany \\ ${ }^{7}$ Department of Surgery, UKSH, Lübeck, Germany \\ ${ }^{8}$ Fraunhofer MEVIS, Institute for Digital Medicine, Bremen, Germany \\ sonja.jaeckle@mevis.fraunhofer.de
}

In endovascular aortic repair (EVAR) procedures fluoroscopy and conventional digital subtraction angiography are currently used to guide the medical instruments inside the patient. Drawbacks of these methods are X-ray exposure and the usage of contrast agents. Moreover, the fluoroscopy provides only a $2 \mathrm{D}$ view, which makes the guidance more difficult. For this reason, a catheter prototype including an optical fiber for shape sensing and three electromagnetic (EM) sensors, which provide the position and orientation information, was built to enable a $3 \mathrm{D}$ catheter guidance. In addition, a model was introduced to process the input data from the tracking systems to obtain the located 3D shape of the first $38 \mathrm{~cm}$ of the catheter [1]. First, a spatial calibration between the optical fiber and each EM sensor was made. This relationship can be used to locate the reconstructed shape of the catheter with the EM sensor poses by determining a rigid transformation. The shape localization method was evaluated using the catheter prototype in different shape measurements and the located 3D shapes were compared with the segmented shapes of the computed tomography scans. The experiments resulted in average errors from 0.99 to $2.29 \mathrm{~mm}$ and maximum errors from 1.73 to $2.99 \mathrm{~mm}$. The accuracies are promising for using the 3D guidance based on an optical fiber and three EM sensors for EVAR procedures. Future work will be to reduce the necessary EM sensors for locating the shape of the catheter. Moreover, this catheter guidance method will be evaluated in a more realistic setting on vessel phantoms.

\section{References}

1. Jäckle S, García-Vázquez V, von Haxthausen F, et al. 3D catheter guidance including shape sensing for endovascular navigation. Proc SPIE. 2020;. 